\title{
Hyperresponsiveness of human gingival fibroblasts from patients with aggressive periodontitis against bacterial lipopolysaccharide
}

\author{
XUE LI, XIAOXUAN WANG and QING-XIAN LUAN \\ Department of Periodontology, Peking University School and Hospital of Stomatology, \\ National Engineering Laboratory for Digital and Material Technology of Stomatology, \\ Beijing Key Laboratory of Digital Stomatology, Beijing 100081, P.R. China
}

Received July 1, 2020; Accepted October 20, 2020

DOI: $10.3892 /$ etm.2021.9861

\begin{abstract}
The present study aimed to investigate whether gingival fibroblasts (GFs) of patients with aggressive periodontitis (AgP) are more sensitive to lipopolysaccharide (LPS) stimulation than GFs of control subjects. AgP causes rapid periodontal destruction, including the production of cytokines [i.e. interleukin (IL)-1 $\beta$, IL-6 and tumor necrosis factor (TNF)- $\alpha$ ] and matrix metalloproteinases (MMP)-1, -3 and -9 in AgP GFs. LPS upregulates IL-1 $\beta$, IL-6, TNF- $\alpha$, MMP-1, MMP-3, MMP-9 and mitochondrial reactive oxygen species (mtROS). Fibroblasts are known to be associated with immune responses to bacterial virulence factors, but the precise mechanisms underlying this severe periodontal disease are unclear. In the present study, primary human GFs of four patients with $\mathrm{AgP}$ and four healthy subjects were challenged in vitro with LPS from Porphyromonas gingivalis (P. gingivalis). The generation of mtROS in GFs was assessed using MitoSOX Red. The expression of genes encoding inflammatory cytokines and MMPs in GFs was analyzed using reverse transcription-quantitative polymerase chain reaction, and the expression of proteins was analyzed using ELISA and Western blotting. Human GFs of patients with AgP exhibited higher
\end{abstract}

Correspondence to: Professor Qing-Xian Luan, Department of Periodontology, Peking University School and Hospital of Stomatology, National Engineering Laboratory for Digital and Material Technology of Stomatology, Beijing Key Laboratory of Digital Stomatology, 22 Zhongguancun Avenue South, Haidian, Beijing 100081, P.R. China E-mail: kqluanqx1100@163.com

Abbreviations: AgP, aggressive periodontitis; GF, gingival fibroblasts; LPS, lipopolysaccharide; IL-1 $\beta$, interleukin-1 $\beta$; mtROS, mitochondrial reactive oxygen species; MMP, matrix metalloproteinase; AGF, human GFs of patients with aggressive periodontitis; HGF, human GFs; AAP, American Academy of Periodontology; ACTB, $\beta$-actin.

Key words: gingival fibroblasts, aggressive periodontitis, lipopolysaccharide, inflammatory response, mtROS, Porphyromonas gingivalis levels of mtROS, and higher mRNA and protein expression levels of proinflammatory cytokines, including IL-1 $\beta$, IL-6, MMP-1, MMP-3 and MMP-9 compared with healthy human GFs following stimulation with LPS from $P$. gingivalis. In the present study, it was demonstrated that GFs of patients with AgP display hyperreactivity when challenged with LPS.

\section{Introduction}

Periodontitis is one of the leading causes of tooth loss in the adult population. This disease can be classified into various categories, and one of the most destructive amongst them is aggressive periodontitis (AgP). The incidence of $\mathrm{AgP}$ is lower than that of other types of periodontitis. However, it affects young individuals and may cause severe destruction of tooth-supporting structures, including tooth loss if left untreated. The current classification for diagnosing periodontal disease was established by the American Academy of Periodontology (AAP) in 1999. This classification provided strict guidelines to aid in $\mathrm{AgP}$ diagnosis. These include three main factors: Systemically healthy individual, rapid loss of clinical attachment and familial aggregation. AgP is characterized by generalized or localized extreme periodontal damage. It is a type of rapid and severe periodontal disease affecting systemically healthy individuals. This disease is characterized by an early age of onset, rapid rate of disease progression and aggregation of family (1).

Fibroblasts serve an important role in chronic infections, including periodontitis. Human gingival fibroblast (GF) is a functionally distinct type of fibroblast in periodontal tissues (2). GFs are subepithelial and predominantly involved in the maintenance and regeneration of periodontal tissues $(3,4)$. The inflammatory destruction in periodontal diseases may be the result of the interactions between bacterial virulence factors and host defense mechanism $(5,6)$. GFs respond to periodontopathic organisms or their components by initiating an inflammatory response, including the production of various proinflammatory cytokines such as interleukin (IL)-1, IL-6 and tumor necrosis factor (TNF)- $\alpha$ (7-9). The results of our previous study demonstrated that GFs may produce mitochondrial reactive oxygen species (mtROS) following stimulation 
with lipopolysaccharides (LPS) of Porphyromonas gingivalis ( $P$. gingivalis) on the tooth surface, which mediates the production of IL-1, IL-6 and TNF- $\alpha$ in GFs (10). Furthermore, the increased production of matrix metalloproteinases (MMPs), including MMP-1, MMP-3 and MMP-9, which degrade numerous extracellular matrix components, may be the cause of GFs contributing toward periodontal tissue destruction $(11,12)$.

Among periodontopathic organisms, $P$. gingivalis is an anaerobic Gram-negative bacterium that appears to be associated with AgP. LPS, located on the outer membrane of the cell wall of Gram-negative bacteria, is an important bacterial surface component, and is considered to be a potent immunostimulant (13). Although periodontitis can be caused by bacteria such as $P$. gingivalis, host susceptibility is crucial to the development of the disease. The characteristics of the cells interacting with LPS from $P$. gingivalis may partly determine susceptibility. For example, mononuclear cells, neutrophils and platelets in patients with periodontitis are known to be different from the same cells in healthy individuals in their interaction with periodontal pathogens (14-18). However, little is known regarding whether differences also exist in GFs between patients with AgP and healthy subjects.

Current evidence has suggested that AgP occurs in susceptible individuals who have abnormal inflammatory/immune response to periodontal pathogens (19). Earlier studies have demonstrated that the expression levels of destruction factors associated with disease progression were higher in periodontitis tissues in patients with chronic periodontitis than in healthy subjects $(20,21)$. At present, there is a lack of data available for comparison of cytokine profiles between GFs obtained from patients with AgP and healthy subjects. The present study tested the hypothesis that GFs in patients with AgP may be more sensitive to LPS stimulation than GFs in control subjects. The present study may elucidate the underlying mechanisms contributing toward the rapid rate of disease progression of AgP.

\section{Materials and methods}

The present study was approved by the Review Board and Ethics Committee of Peking University Health Science Center. Written informed consent was obtained from all subjects.

Cell culture and treatment. GFs derived from four patients with AgP (AGFs) and healthy subjects, who sought dental treatment at the Department of Periodontology, Peking University School and Hospital of Stomatology between January 2017 and January 2018, were used in the present study. The test group consisted of 2 males and 2 females, aged 38-50 years with a mean age of 44 . The control group consisted of 2 males and 2 females, aged 32 to 40 years with a mean age of 36. Exclusion criteria included pregnancy or breastfeeding, smoking, alcohol abuse, uncontrolled diabetes and other systemic conditions that could affect the periodontal status. Healthy GFs (CGFs) were obtained from explants of human normal gingival tissues as a control group from patients seeking dental treatment at the Department of Periodontology, Peking University School and Hospital of Stomatology between January 2017 and January 2018 (Table I; Fig. 1). GF explantation was performed as previously described (22). Individuals were designated as having $\mathrm{AgP}$ according to the 1999 AAP Classification of Periodontal Diseases (1). Healthy gingival tissue samples were collected from periodontal healthy groups undergoing a crown-lengthening procedure, while inflamed gingival tissue was harvested from teeth with pockets of $6 \mathrm{~mm}$ or more in patients with chronic periodontitis following flap debridement (22). The cells were cultured at $37^{\circ} \mathrm{C}$ and $5 \% \mathrm{CO}_{2}$ in Dulbecco's modified Eagle's medium (DMEM; HyClone; Cytiva), containing 10\% (v/v) fetal bovine serum (FBS; HyClone; Cytiva) and $100 \mathrm{U} / \mathrm{ml}$ penicillin with $100 \mu \mathrm{g} / \mathrm{ml}$ streptomycin. GFs between passages 3-6 were used. The cells were treated with $1 \mu \mathrm{g} / \mathrm{ml}$ LPS derived from $P$. gingivalis (InvivoGen, cat. no. tlrl-pglps) for $12 \mathrm{~h}$. The experiments on the $4 \mathrm{AgP}$ patients and 4 healthy control subjects were repeated 5 times.

Multimodal microplate reader. Fluorescence was measured using a multimodal microplate reader (BioTek Instruments, Inc.). HGFs were trypsinized and washed with cold phosphate-buffered saline (PBS). The cells $\left(1.8 \times 10^{5}\right)$ were resuspended in $1 \mathrm{ml}$ DMEM containing $5 \mu \mathrm{M}$ MitoSOX Red and incubated in the dark in $\mathrm{CO}_{2}$ incubator for $30 \mathrm{~min}$. The cells were centrifuged at $130 \mathrm{x} \mathrm{g}$ for $5 \mathrm{~min}$ at room temperature, washed three times with PBS, and resuspended in $500 \mu \mathrm{l}$ PBS. The mtROS content of cells was analyzed based on the fluorescence intensity of MitoSOX Red. The mtROS content of cells was analyzed based on the fluorescence intensity of MitoSOX Red with excitation at $510 \mathrm{~nm}$ and emission at $580 \mathrm{~nm}$.

RNA isolation and reverse transcription-quantitative polymerase chain reaction (RT-qPCR). HGFs and AGFs were cultured in 6 -well plates $\left(1 \times 10^{5}\right.$ per well) followed by the addition of medium with or without LPS. Total RNA was isolated using TRIzol ${ }^{\circledR}$ reagent (Invitrogen; Thermo Fisher Scientific, Inc.) according to the manufacturer's protocol. cDNA was synthesized using Reverse Transcription Premix (Bioneer Corporation). The thermal profile was incubation at $70^{\circ} \mathrm{C}$ for $5 \mathrm{~min}$ before chilling on ice. PCR was performed using gene-specific primers (Table II) and PCR premix (Kapa Biosystems; Roche Diagnostics). The PCR conditions were $10 \mathrm{~min}$ at $95^{\circ} \mathrm{C}$, followed by 40 cycles of $95^{\circ} \mathrm{C}$ for $15 \mathrm{sec}$ and $60^{\circ} \mathrm{C}$ for $60 \mathrm{sec}$ (PCR machine model: Eppendorf Mastercycler X50h; Eppendorf). All reactions were performed in triplicate in two separate experiments. The relative expression levels of the targets in each sample were calculated using the comparative $2^{-\Delta \Delta \mathrm{Cq}}$ method following normalization against the expression of ACTB (21).

Measurement of various cytokine levels by ELISA. The GFs were cultured in 96 -well plates $\left(1 \times 10^{4}\right.$ per well) and then medium with or without $1 \mu \mathrm{g} / \mathrm{ml}$ LPS was added. The levels of IL-1 $\beta$, IL- 6 and TNF- $\alpha$ in cell culture supernatants were measured using ELISA kits (R\&D Systems China Co., Ltd.; cat. no. IL-1 $\beta$, 70-E-EK101B1; IL-6, 70-E-EK1061; TNF- $\alpha$, EK1821), according to the manufacturer's protocols.

Western blotting. Whole protein lysates were prepared using PRO-PREP Protein Extraction Solution (Intron 
Table I. Demographic characteristics of the study sample at baseline.

\begin{tabular}{|c|c|c|c|c|}
\hline Sample & Age, years & Sex & Location & Treatment \\
\hline Patient 1 & 38 & Female & $15,16,17$ & Flap debridement \\
\hline Patient 2 & 43 & Female & $24,25,26,27$ & Flap debridement \\
\hline Patient 3 & 45 & Male & 26,27 & Flap debridement \\
\hline Patient 4 & 50 & Male & $44,45,46,47$ & Flap debridement \\
\hline Healthy control 1 & 34 & Male & 16 & Crown-lengthening \\
\hline Healthy control 2 & 40 & Female & 36 & Crown-lengthening \\
\hline Healthy control 3 & 38 & Male & 25 & Crown-lengthening \\
\hline Healthy control 4 & 32 & Female & 26 & Crown-lengthening \\
\hline
\end{tabular}

Table II. Primers used for gene amplification.

\begin{tabular}{llll}
\hline Gene & Primer name & \multicolumn{1}{c}{ Primer sequence } & Primer length $(\mathrm{bp})$ \\
\hline IL-1 $\beta$ & IL-1 $\beta$-forward & CTTCAGCCAATCTTCATTGCT & 200 \\
IL-1 $\beta$-reverse & TCGGAGATTCGTAGCTGGAT & GAGGGCTCTTCGGCAAATGTA \\
IL-6-forward & IL-6-reverse & CCCAGTGGACAGGTTTCTGAC \\
TNF- $\alpha$ & TNF- $\alpha$-forward & GCTCAGACATGTTTTCCGTGAA \\
MMP-1 & MMP-1-forward & GTCACCAAATCAGCATTGTTTAGA \\
& MMP-1-reverse & TCTGGGGAAAACCTTTCGACT & 89 \\
MMP-3 & MMP-3-forward & CACCAACGTATTCAAAAGCACAA & 140 \\
MMP-9 & MMP-3-reverse & AGTCTTCCAATCCTACTGTTGCT \\
& MMP-9-forward & TCCCGTATGGTTACACCAATCC \\
ACTB & MMP-9-reverse & TGTACCGCTATGGTTACACTCG & 136 \\
& ACTB-forward & GGCAGGGACAGTTGCTTCT & 180 \\
\hline
\end{tabular}

IL, interleukin; TNF- $\alpha$, tumor necrosis factor- $\alpha$; MMP, matrix metalloproteinase; ACTB, $\beta$-actin.

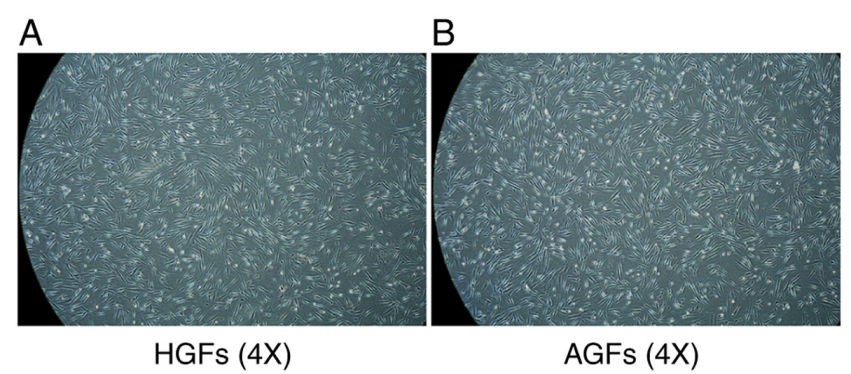

Figure 1. Images of typical morphology of GFs from AgP and from healthy subjects. (A) HGFs of passage 0. (B) AGFs of passage 0. GF, gingival fibroblasts; AgP, aggressive periodontitis; HGFs, GFs from healthy subjects; AGFs, GFs from patients with aggressive periodontitis.

Biotechnology, Inc.). The nuclear and cytoplasmic fractions were isolated using an NE-PER Nuclear and Cytoplasmic Extraction Reagent kit (Thermo Fisher Scientific, Inc.), according to the manufacturer's protocol. Equal amounts (15 $\mu \mathrm{l})$ of protein, measured using the BCA protein assay (Pierce; Thermo Fisher Scientific, Inc.) were loaded into each lane of the gel. Proteins were separated by electrophoresis on $10-12 \%(\mathrm{v} / \mathrm{v})$ sodium dodecyl sulphate-polyacrylamide gels and transferred onto nitrocellulose membranes (BD Biosciences). The membranes were then blocked with 5\% BSA (Beijing Solarbio Science \& Technology Co., Ltd.; cat. no. SW3015) for $2 \mathrm{~h}$ at room temperature and incubated with rabbit antibodies against MMP-1 (cat. no. Ab137332; Abcam), MMP-3 (cat. no. Ab2915; Abcam), MMP-9 (cat. no. Ab38898; Abcam) and actin (cat. no. TA-09; Abcam) at a 1:1,000 dilution for $2 \mathrm{~h}$ at room temperature. The membranes were then incubated with horseradish peroxidase (HRP)-coupled anti-rabbit secondary antibody (Cell Signaling Technology, Inc. cat. no. 5151, 1:2,000) at room temperature for $40 \mathrm{~min}$. The membranes were then washed with TBST (20\% Tween-20) 3 times, for 10 min each time. Finally, the bands were stained with an enhanced chemiluminescence kit (Thermo Fisher Scientific, Inc.). Rabbit anti-actin was used as a loading control (Cell Signaling Technology, Inc.). After the image was scanned, grayscale analysis was performed using Gel Image system ver.4.00 (Tanon Science and Technology Co., Ltd.). 
A

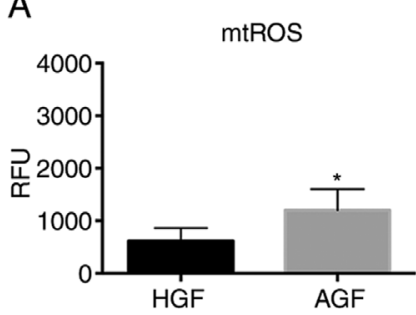

B

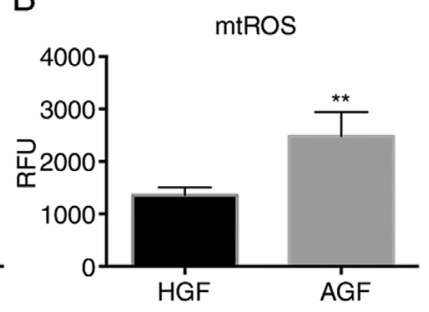

Figure 2. Level of mtROS in GFs in control subjects and patients with AgP prior to and following a $12 \mathrm{~h}$ challenge with LPS from P. gingivalis. (A) The level of mtROS in GFs of control subjects and patients with AgP prior to LPS challenge. (B) The level of mtROS in GFs of control subjects and patients with AgP following LPS challenge. ${ }^{*} \mathrm{P}<0.05,{ }^{* *} \mathrm{P}<0.01$ vs. HGF. mtROS, mitochondrial reactive oxygen species; GFs, gingival fibroblasts; $\mathrm{AgP}$, aggressive periodontitis; LPS, lipopolysaccharide; HGFs, GFs from healthy subjects; AGFs, GFs from patients with aggressive periodontitis; RFU, relative fluorescence units.

Statistical analysis. Data represent the mean \pm standard deviation of five independent experiments. Data were analyzed using GraphPad Prism software (version 6; GraphPad Software, Inc.). Variance between the two groups was analyzed by t-test. $\mathrm{P}<0.05$ was considered to indicate a statistical difference. $\mathrm{P}<0.01$ was considered to indicate a statistically significant difference.

\section{Results}

Comparison of morphology of CGFs and AGF. The images of typical morphology of GFs from patients with AgP and from healthy subjects were observed under a microscope (Fig. 1). No difference in morphology or proliferation was identified between CGFs and AGFs.

Comparison of basal levels and augmented mtROS levels stimulated by LPS in CGFs and AGFs. The levels of mtROS in unstimulated or stimulated GFs were measured and were compared between the heathy and the AgP groups (Fig. 2). In the unstimulated cells, the mtROS level was higher in the AgP group compared with the healthy group $(\mathrm{P}<0.05)$. In the stimulated cells, the mtROS level was significantly higher in the $\mathrm{AgP}$ group than in the healthy group $(\mathrm{P}<0.01)$.

Comparison of mRNA expression levels of proinflammatory genes in HGFs and AGF prior to and following stimulation by LPS. The mRNA expression levels (relative to the housekeeping gene) of IL-1 $\beta$, IL- 6 , and TNF- $\alpha$, which encode proinflammatory cytokines in GFs, were measured in unstimulated or stimulated GFs. These levels were then compared between the heathy and the AgP groups (Fig. 3). In the unstimulated cells, IL-1 $\beta$ and IL-6 mRNA expression was higher in the $\mathrm{AgP}$ group compared with the healthy group (IL-1 $\beta, \mathrm{P}<0.05$; IL-6, P<0.05; Fig. $3 \mathrm{~A}$ and $\mathrm{C}$ ), while the expression of TNF- $\alpha$ mRNA did not show a significant difference between the healthy and $\operatorname{AgP}$ groups (TNF- $\alpha, \mathrm{P}>0.05$; Fig. 3E). In stimulated cells, the expression level of IL-1 $\beta$ and IL-6 mRNA was significantly higher in the AgP group than in the healthy group ( $\mathrm{P}<0.01$; Fig. 3B and $\mathrm{D})$, and TNF- $\alpha$ mRNA was higher in the AgP group than in the healthy group (TNF- $\alpha$, P $<0.05$; Fig. 3F).
A

IL-1 $\beta$

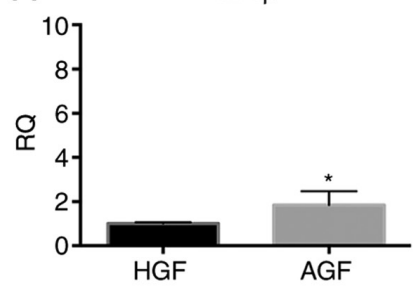

B

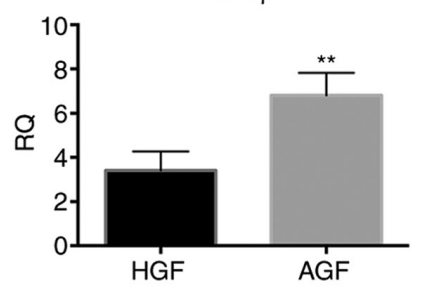

C

IL-6

D
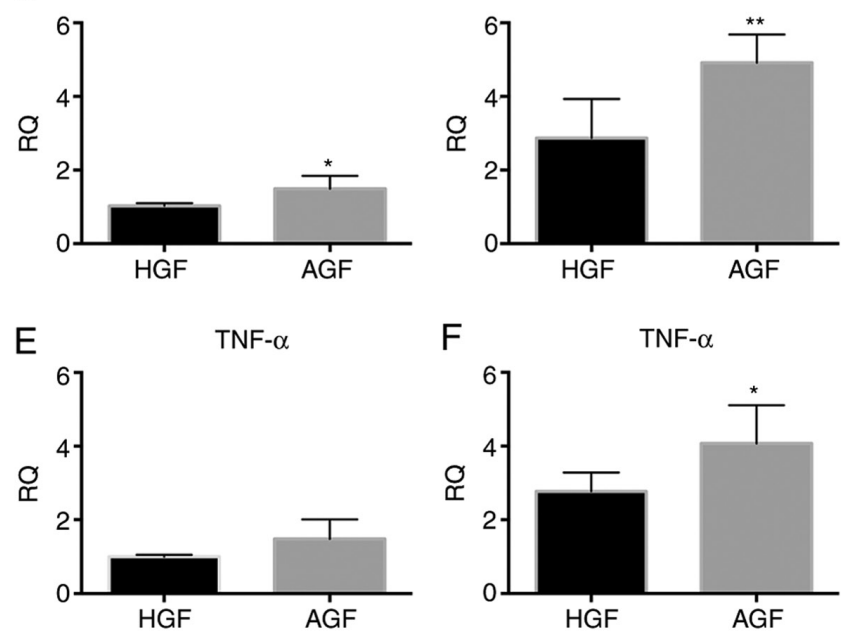

$\mathrm{F}$

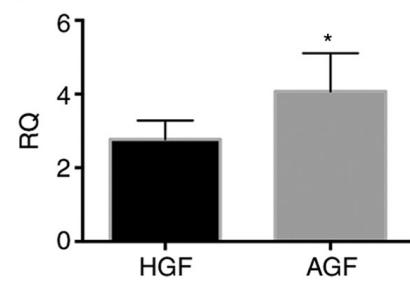

Figure 3. mRNA expression of proinflammatory cytokines, including IL-1 $\beta$, IL-6, TNF- $\alpha$ in control subjects and patients with AgP prior to and following a 12 h challenge with LPS from $P$. gingivalis. The mRNA expression of IL-1 $\beta$ in GFs of control subjects and patients with $\operatorname{AgP}(\mathrm{A})$ prior to LPS challenge and (B) following LPS challenge. The mRNA expression of IL-6 in GFs of control subjects and patients with $\operatorname{AgP}(\mathrm{C})$ prior to LPS challenge and (D) following LPS challenge. The mRNA expression of TNF- $\alpha$ in GFs of control subjects and patients with $\mathrm{AgP}(\mathrm{E})$ prior to LPS challenge and (F) following LPS challenge. ${ }^{*} \mathrm{P}<0.05,{ }^{* *} \mathrm{P}<0.01$ vs. HGF. IL, interleukin; TNF- $\alpha$, tumor necrosis factor- $\alpha$; GFs, gingival fibroblasts; AgP, aggressive periodontitis; LPS, lipopolysaccharide; HGFs, GFs from healthy subjects; AGFs, GFs from patients with aggressive periodontitis; RQ, relative quantity.

Comparison of protein levels of proinflammatory cytokines in HGFs and AGF prior to and following LPS stimulation. To analyze whether changes in cytokines gene expression also resulted in increased protein secretion, culture supernatants of challenged and unchallenged GFs of patients and control subjects were analyzed for the presence and levels of IL-1 $\beta$, IL-6 and TNF- $\alpha$ (Fig. 4). IL-1 $\beta$ and IL-6 levels in GFs unstimulated with LPS were higher in the AgP group than in the healthy group (IL-1 $\beta, \mathrm{P}<0.05$; IL-6, $\mathrm{P}<0.05$ Fig. $4 \mathrm{~A}$ and $\mathrm{C}$ ). However, the expression of TNF- $\alpha$ did not significantly differ between the healthy and AgP groups (TNF- $\alpha, \mathrm{P}>0.05$; Fig. 4E). With stimulation, IL-1 $\beta$, IL- 6 and TNF- $\alpha$ levels in GFs were significantly higher in the $\mathrm{AgP}$ group than in the healthy group (IL-1 $\beta, \mathrm{P}<0.01$; IL-6, P<0.01; TNF- $\alpha, \mathrm{P}<0.01$; Fig. 4B, $\mathrm{D}$ and $\mathrm{F})$. There was a difference in the ratio of cytokines between GFs from AgP and GFs from healthy subjects when compared the unstimulated with stimulated states. Under the stimulated state, the ratio was higher in the $\mathrm{AgP}$ group than in the healthy group (IL-1 $\beta, \mathrm{P}<0.05$; IL-6, $\mathrm{P}<0.05$; TNF- $\alpha$, $\mathrm{P}<0.05$; Fig. S1A-C). degradation in HGFs and AGF with and without LPS 

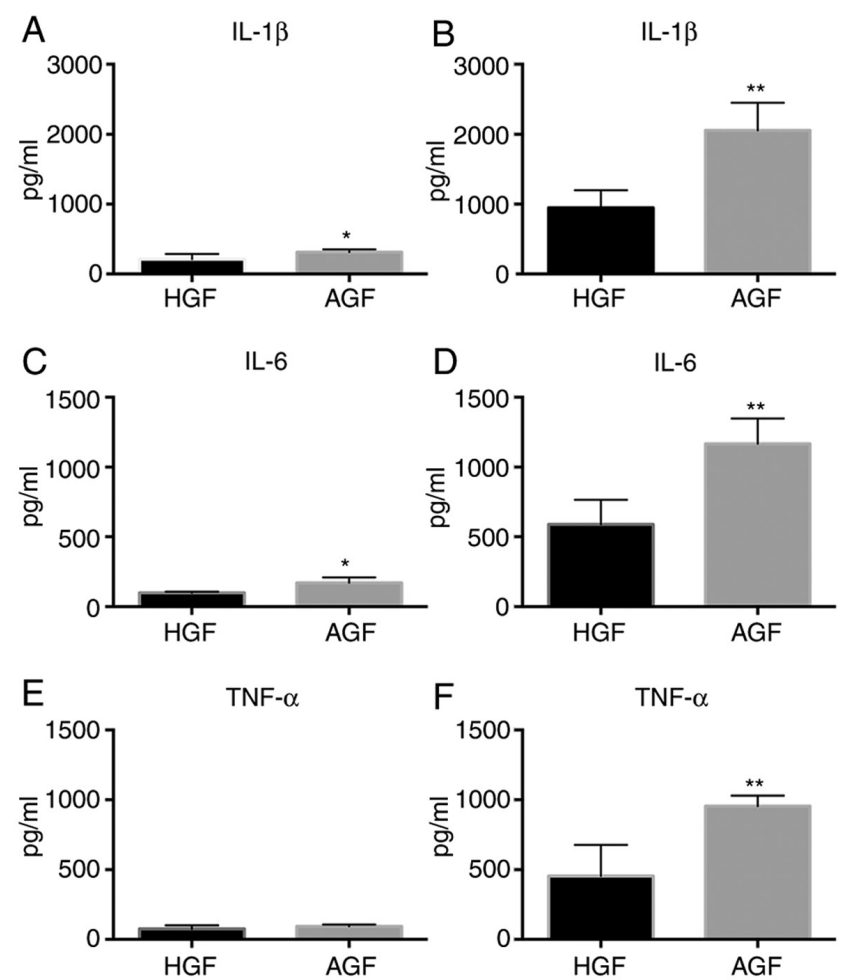

Figure 4. Protein expression of proinflammatory cytokines, including IL-1 $\beta$, IL-6 and TNF- $\alpha$ in control subjects and patients with AgP prior to and following $12 \mathrm{~h}$ challenge with LPS from $P$. gingivalis. Protein expression of IL-1 $\beta$ in GFs of control subjects and patients with AgP (A) prior to LPS challenge and (B) following LPS challenge. Protein expression of IL-6 in GFs of control subjects and patients with $\mathrm{AgP}(\mathrm{C})$ prior to LPS challenge and (D) following LPS challenge. Protein expression of TNF- $\alpha$ in GFs of control subjects and patients with $\mathrm{AgP}(\mathrm{E})$ prior to LPS challenge and $(\mathrm{F})$ following LPS challenge. ${ }^{*} \mathrm{P}<0.05,{ }^{* * *} \mathrm{P}<0.01$ vs. HGF. IL, interleukin; TNF- $\alpha$, tumor necrosis factor- $\alpha$; GFs, gingival fibroblasts; AgP, aggressive periodontitis; LPS, lipopolysaccharide; HGFs, GFs from healthy subjects; AGFs, GFs from patients with aggressive periodontitis.

stimulation. Gene expression of MMP-1, MMP-3 and MMP-9 in unstimulated or stimulated GFs was compared between the healthy and the AgP groups (Fig. 5). In the unstimulated cells, MMP-1, MMP-3 and MMP-9 mRNA expression was higher in the AgP group than in the healthy group (MMP-1, $\mathrm{P}<0.05$; MMP-3, $\mathrm{P}<0.05$; MMP-9, $\mathrm{P}<0.05$; Fig. 5A, $\mathrm{C}$ and $\mathrm{E}$ ). In the stimulated cells, MMP-1, MMP-3 and MMP-9 mRNA expression was significantly higher in the $\mathrm{AgP}$ group than in the healthy group (MMP-1, P<0.01; MMP-3, P<0.01; MMP-9, $\mathrm{P}<0.01$; Fig. 5B, D and F).

Comparison of protein levels of genes associated with matrix degradation in GFs of HGFs and AGF with and without LPS stimulation. To investigate whether the difference was also present at the protein levels, the protein expression of MMP-1, MMP-3 and MMP-9 was measured through Western blotting. In the unstimulated cells, the levels of MMP-1 and MMP-9 were higher in the AgP group than in the healthy group $(\mathrm{P}<0.05$; Fig. 6A-C), but there was no difference in MMP-3 expression between $\mathrm{AgP}$ and healthy groups ( $\mathrm{P}>0.05$; Fig. $6 \mathrm{~A}$ and $\mathrm{D})$. In the stimulated cells, the protein expression of MMP-1, MMP-3 and MMP-9 was significantly higher in the AgP group than in the healthy group (MMP-1, $\mathrm{P}<0.01$; MMP-3, $\mathrm{P}<0.01$; MMP-9, $\mathrm{P}<0.01$; Fig. 6A-D).
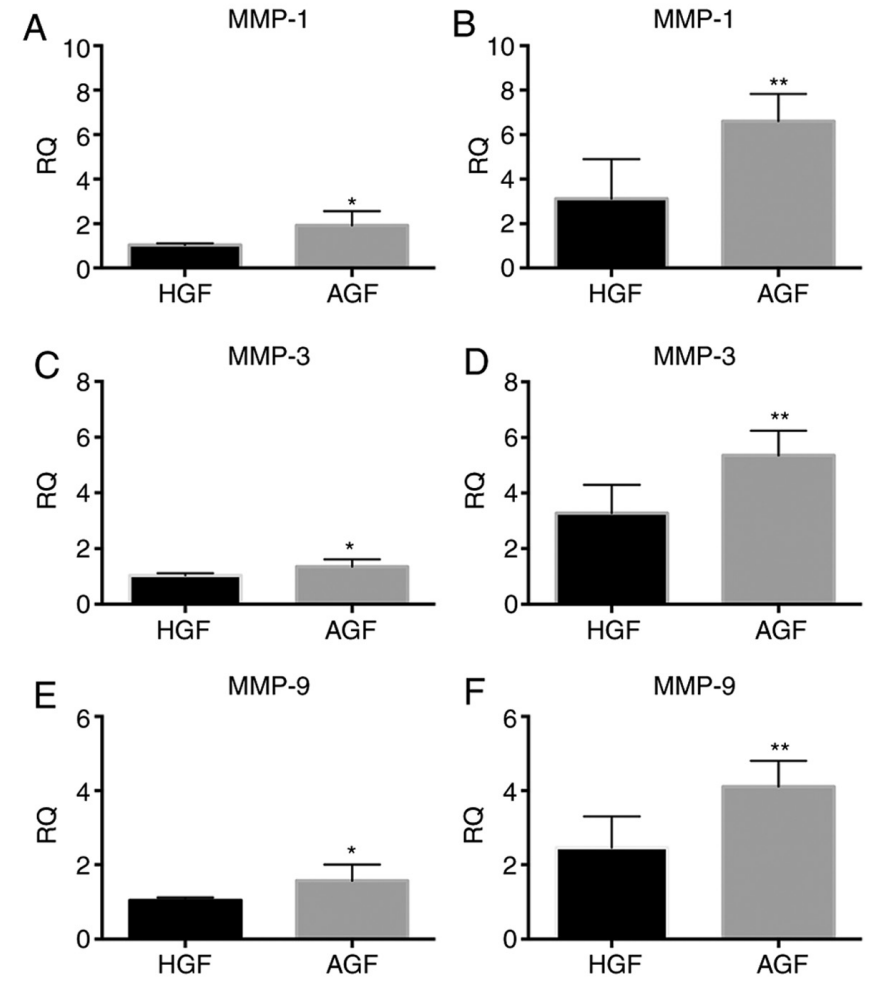

Figure 5. mRNA expression levels of MMP-1, MMP-3 and MMP-9, in control subjects and patients with $\mathrm{AgP}$ prior to and following $12 \mathrm{~h}$ challenge with LPS from $P$. gingivalis. The mRNA expression of MMP-1 in GFs of control subjects and patients with $\operatorname{AgP}(\mathrm{A})$ prior to LPS challenge and (B) following LPS challenge. The mRNA expression of MMP-3 in GFs of control subjects and patients with $\mathrm{AgP}(\mathrm{C})$ prior to LPS challenge and (D) following LPS challenge. The mRNA expression of MMP-9 in GFs of control subjects and patients with $\mathrm{AgP}$ (E) prior to LPS challenge and (F) following LPS challenge. ${ }^{*} \mathrm{P}<0.05,{ }^{* *} \mathrm{P}<0.01$ vs. HGF. MMP, matrix metalloproteinases; AgP, aggressive periodontitis; LPS, lipopolysaccharide; GFs, gingival fibroblasts; HGFs, GFs from healthy subjects; AGFs, GFs from patients with aggressive periodontitis; RQ, relative quantity.

\section{Discussion}

Human GFs are the most abundant resident cells in periodontal tissue. The continuous expression of inflammatory cytokines by GFs may be involved in the overproduction of lytic enzymes, apoptotic factors and bone-resorbing mediators, resulting in periodontal tissue destruction. Excessive production of these mediators is important for the pathogenesis and progression of periodontitis.

In the present study, it was hypothesized that as the host response is important in the pathogenesis of AgP, GFs respond differently to LPS challenge in patients with $\mathrm{AgP}$ than in healthy controls. Therefore, GFs of patients with AgP and healthy control subjects were challenged with LPS from $P$. gingivalis.

In the present study, AGFs exhibited higher expression of IL-1 $\beta$, IL-6, MMP-1, MMP-3 and MMP-9 without LPS than HGFs. It was not possible to verify the difference in TNF- $\alpha$ mRNA expression in GFs between patients with AgP and control subjects without LPS. After 12-h LPS stimulation, AGFs exhibited higher expression of IL-1 $\beta$, IL- 6 , TNF- $\alpha$, MMP-1, MMP-3 and MMP-9 than HGFs. A previous study reported that the comparison of mRNA and protein expression of inflammatory genes was higher in inflamed GFs (IGFs; 

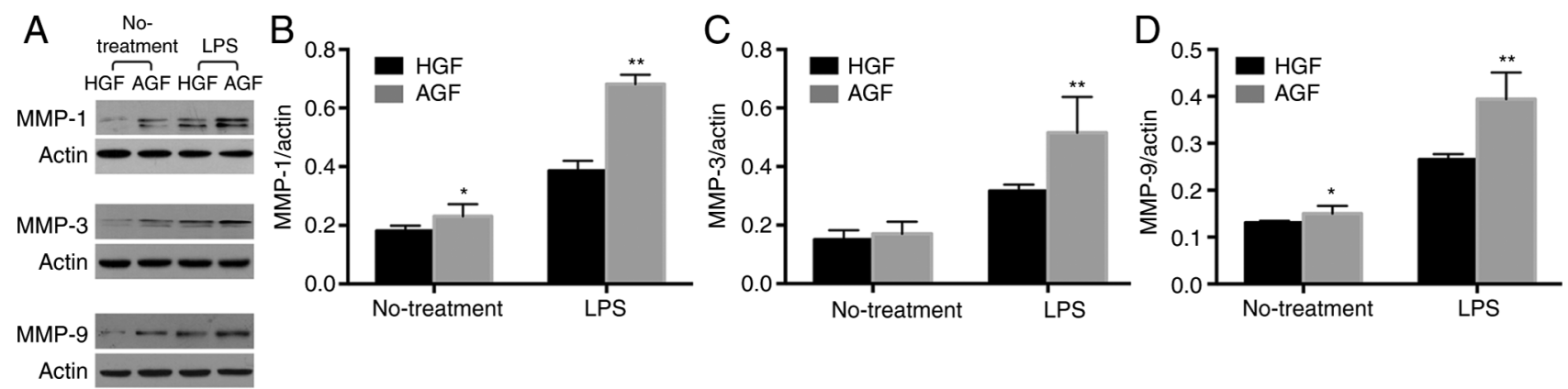

Figure 6. Protein levels of MMP-1, MMP-3 and MMP-9, in control subjects and patients with AgP prior to and following $12 \mathrm{~h}$ challenge with LPS from P. gingivalis. (A) The results of Western blotting of MMP-1, MMP-3 and MMP-9 in GFs of control subjects and patients with AgP prior to and following LPS challenge. The analysis of the relative content of (B) MMP-1, (C) MMP-3 and (D) MMP-9. ${ }^{*} \mathrm{P}<0.05,{ }^{* *} \mathrm{P}<0.01$ vs. treatment matched HGF. MMP, matrix metalloproteinases; AgP, aggressive periodontitis; LPS, lipopolysaccharide; GFs, gingival fibroblasts; HGFs, GFs from healthy subjects; AGFs, GFs from patients with aggressive periodontitis.

GFs isolated from patients with chronic periodontitis) than in HGFs after stimulation with P. gingivalis LPS (21). The present study was consistent with this previous report $(21,23)$. To date, few studies have focused on AGFs and the present study was the first to employ $P$. gingivalis LPS to stimulate AGFs and compare the different responses in HGFs. $P$. gingivalis LPS mediates inflammation by inducing the release of proinflammatory cytokines in HGFs (24). The present study identified that the expression of inflammatory cytokines and MMPs was elevated following challenge with $P$. gingivalis LPS in AGFs. Furthermore, the differences in factors associated with inflammation were identified in GFs between the $\mathrm{AgP}$ and the healthy groups with/without LPS stimulation. Additionally, the differences in GFs between the $\mathrm{AgP}$ and the healthy groups with LPS stimulation were more significant. The results of the present study demonstrated that GFs of patients with $\mathrm{AgP}$ exhibit hyperreactivity in the presence of LPS. The present study has another limitation in that expression was analyzed at only one time point. It may take more time for differences in cytokine and MMP responses to become detectable. In addition to the inflammatory cytokines and MMPs, mtROS was higher in GFs in patients with AgP than in control subjects in unchallenged and challenged cells. LPS-stimulated AGFs produced inflammatory cytokines more significantly than HGFs.

MtROS are produced in the process of normal aerobic cell metabolism, serve important physiological roles in maintaining cell redox status, and are necessary for normal cellular function. They are generated as by-products of energy production, depending on the normal structure and function of mitochondrion (25). More mtROS are generated in AGFs, suggesting a possible dysfunction or a morphological change in mitochondria (26). Mitochondrial dysfunction may increase allergic airway inflammation (27), and increase inflammatory response to cytokines in normal human chondrocytes (28). In the present study, AGFs exhibited a marked increase in LPS-triggered activation of inflammatory cytokines, accompanied by MMPs release. Whether the response of GFs in AgP patients to LPS is aggravated by the increased mtROS will be a focus of our future studies.

In conclusion, the present study demonstrated that GFs of patients with $\mathrm{AgP}$ display hyperreactivity when challenged with LPS. Although in vivo analyses are required to verify these findings, these results explain, in part, the difference in cellular responses between patients with $\mathrm{AgP}$ and healthy subjects. The results of the present study may help aid understanding of the pathogenesis of $\mathrm{AgP}$ and development of novel strategies to alleviate the inflammation. There is no clear evidence of a causal association between mtROS and aggressive periodontitis. However, increasing data have suggested that it may be an important factor in the pathogenesis of the disease.

\section{Acknowledgements}

Not applicable.

\section{Funding}

The present study was funded by the National Natural Science Foundation of China (grant no. 81271148).

\section{Availability of data and materials}

The datasets used and/or analyzed during the current study are available from the corresponding author on reasonable request.

\section{Authors' contributions}

QXL designed the study; XL and XW performed the experiments, analyzed the data and prepared the manuscript. QXL reviewed the manuscript. All authors read and approved the final manuscript.

\section{Ethics approval and consent to participate}

The present study was approved by the Medical Ethical Committee of the School of Stomatology, Peking University (approval no. PKUSSIRB-2013017).

\section{Patient consent for publication}

Not applicable.

\section{Competing interests}

The authors declare that they have no competing interests. 


\section{References}

1. No authors listed: 1999 International International Workshop for a classification of periodontal diseases and conditions. Papers. Oak Brook, Illinois, October 30-November 2, 1999. Ann Periodontol 4: i, 1-112, 1999.

2. Buckley CD, Pilling D, Lord JM, Akbar AN, Scheel-Toellner D and Salmon M: Fibroblasts regulate the switch from acute resolving to chronic persistent inflammation. Trends Immunol 22: 199-204, 2001.

3. Ara T, Kurata K, Hirai K, Uchihashi T, Uematsu T, Imamura Y, Furusawa K, Kurihara S and Wang PL: Human gingival fibroblasts are critical in sustaining inflammation in periodontal disease. J Periodontal Res 44: 21-27, 2009.

4. Flavell SJ, Hou TZ, Lax S, Filer AD, Salmon M and Buckley CD: Fibroblasts as novel therapeutic targets in chronic inflammation. Br J Pharmacol 153 (Suppl 1): S241-S246, 2008.

5. Dongari-Bagtzoglou AI and Ebersole JL: Increased presence of interleukin-6 (IL-6) and IL-8 secreting fibroblast subpopulations in adult periodontitis. J Periodontol 69: 899-910, 1998.

6. Pathirana RD, O'Brien-Simpson NM and Reynolds EC: Host immune responses to Porphyromonas gingivalis antigens. Periodontol 2000 52: 218-237, 2010.

7. Wilson M: Biological activities of lipopolysaccharides from oral bacteria and their relevance to the pathogenesis of chronic periodontitis. Sci Prog 78: 19-34, 1995.

8. Garlet GP: Destructive and protective roles of cytokines in periodontitis: A re-appraisal from host defense and tissue destruction viewpoints. J Dent Res 89: 1349-1363, 2010.

9. Liu J, Wang Y and Ouyang X: Beyond toll-like receptors: Porphyromonas gingivalis induces IL-6, IL-8, and VCAM-1 expression through NOD-mediated NF-KB and ERK signaling pathways in periodontal fibroblasts. Inflammation 37: 522-533, 2014.

10. Li X, Wang X, Zheng M and Luan QX: Mitochondrial reactive oxygen species mediate the lipopolysaccharide-induced pro-inflammatory response in human gingival fibroblasts. Exp Cell Res 347: 212-221, 2016.

11. Goncalves PF, Huang H, McAninley S, Alfant B, Harrison P, Aukhil I, Walker C and Shaddox LM: Periodontal treatment reduces matrix metalloproteinase levels in localized aggressive periodontitis. J Periodontol 84: 1801-1808, 2013.

12. Seguier S, Gogly B, Bodineau A, Godeau G and Brousse N: Is collagen breakdown during periodontitis linked to inflammatory cells and expression of matrix metalloproteinases and tissue inhibitors of metalloproteinases in human gingival tissue? J Periodontol 72: 1398-1406, 2001.

13. Henderson B, Poole S and Wilson M: Bacterial modulins: A novel class of virulence factors which cause host tissue pathology by inducing cytokine synthesis. Microbiol Rev 60: 316-341, 1996.

14. Gustafsson A, Ito H, Asman B and Bergstrom K: Hyper-reactive mononuclear cells and neutrophils in chronic periodontitis. J Clin Periodontol 33: 126-129, 2006.

15. Nicu EA, Van der Velden U, Everts V, Van Winkelhoff AJ, Roos D and Loos BG: Hyper-reactive PMNs in FcgammaRIIa $131 \mathrm{H} / \mathrm{H}$ genotype periodontitis patients. J Clin Periodontol 34: 938-945, 2007
16. Matthews JB, Wright HJ, Roberts A, Cooper PR and Chapple IL: Hyperactivity and reactivity of peripheral blood neutrophils in chronic periodontitis. Clin Exp Immunol 147: 255-264, 2007.

17. Zhan Y, Lu R, Meng H, Wang X and Hou J: Platelet activation and platelet-leukocyte interaction in generalized aggressive periodontitis. J Leukoc Biol 100: 1155-1166, 2016.

18. Nicu EA, Van der Velden U, Nieuwland R, Everts V and Loos BG: Elevated platelet and leukocyte response to oral bacteria in periodontitis. J Thromb Haemost 7: 162-170, 2009.

19. Ogawa T, Ozaki A, Shimauchi $\mathrm{H}$ and Uchida $\mathrm{H}$ : Hyporesponsiveness of inflamed human gingival fibroblasts from patients with chronic periodontal diseases against cell surface components of Porphyromonas gingivalis. FEMS Immunol Med Microbiol 18: 17-30, 1997.

20. Scheres N, Laine ML, Sipos PM, Bosch-Tijhof CJ, Crielaard W, de Vries TJ and Everts V: Periodontal ligament and gingival fibroblasts from periodontitis patients are more active in interaction with Porphyromonas gingivalis. J Periodontal Res 46: 407-416, 2011.

21. Kang W, Hu Z and Ge S: Healthy and inflamed gingival fibroblasts differ in their inflammatory response to Porphyromonas gingivalis lipopolysaccharide. Inflammation 39: 1842-1852, 2016.

22. Zdarilova A, Svobodova A, Simanek V and Ulrichova J: Prunella vulgaris extract and rosmarinic acid suppress lipopolysaccharide-induced alteration in human gingival fibroblasts. Toxicol In Vitro 23: 386-392, 2009.

23. Wang PL, Ohura K, Fujii T, Oido-Mori M, Kowashi Y, Kikuchi M, Suetsugu Y and Tanaka J: DNA microarray analysis of human gingival fibroblasts from healthy and inflammatory gingival tissues. Biochem Biophys Res Commun 305: 970-973, 2003.

24. Imatani $\mathrm{T}$, Kato $\mathrm{T}$ and Okuda K: Production of inflammatory cytokines by human gingival fibroblasts stimulated by cell-surface preparations of Porphyromonas gingivalis. Oral Microbiol Immunol 16: 65-72, 2001

25. Kowaltowski AJ, de Souza-Pinto NC, Castilho RF and Vercesi AE: Mitochondria and reactive oxygen species. Free Radic Biol Med 47: 333-343, 2009.

26. Yu T, Robotham JL and Yoon Y: Increased production of reactive oxygen species in hyperglycemic conditions requires dynamic change of mitochondrial morphology. Proc Natl Acad Sci USA 103: 2653-2658, 2006.

27. Aguilera-Aguirre L, Bacsi A, Saavedra-Molina A, Kurosky A, Sur S and Boldogh I: Mitochondrial dysfunction increases allergic airway inflammation. J Immunol 183: 5379-5387, 2009.

28. Vaamonde-Garcia C, Riveiro-Naveira RR, Valcarcel-Ares MN, Hermida-Carballo L, Blanco FJ and Lopez-Armada MJ: Mitochondrial dysfunction increases inflammatory responsiveness to cytokines in normal human chondrocytes. Arthritis Rheum 64: 2927-2936, 2012. 